\title{
Influence of acceleration and deceleration of Super Heavy Loads (SHLs) on the service life of pavement structures
}

\author{
Ali Morovatdar \\ Graduate Research Assistant, Department of Civil engineering, University of Texas at El Paso, \\ Texas, USA \\ Reza S. Ashtiani \\ Associate Professor, Department of Civil engineering, University of Texas at El Paso, Texas, USA
}

\begin{abstract}
Super Heavy Load (SHL) vehicles typically consist of heavy axles that impart substantial damages on transportation facilities such highway pavements and bridges. The taxing loading conditions, when combined with acceleration and deceleration forces from SHL vehicles, results in substantial interfacial shear stresses that manifests itself in premature failure of transportation facilities. This was the motivation for our research team to explore the detrimental effects of the acceleration and deceleration on the service life of roadways. To achieve this objective, our research team, initially deployed portable Weight-in-Motion (WIM) devices to ten sites with high frequency of SHLs in overload corridors of east and southeast Texas. Subsequently, nondestructive field tests such as Falling Weight Deflectometer (FWD) and Ground Penetrating Radar (GPR) were performed during summer and winter months for the back-calculation of the layer moduli in ten sites. The field observations and measurements were in turn incorporated in a three-dimensional (3D) finite element code for characterization of the influence of the acceleration and deceleration on the mechanical responses of the pavement structures. Then, a series of scenarios consisted of different patterns of acceleration, and deceleration were simulated in the 3D finite element software for ten sites and the results were contrasted with the steady rolling conditions. The sensitivity analyses of the representative pavements sections in this study showed that the deceleration or braking forces from the SHL vehicles resulted in the development of significant shear stresses in surface layers of thin structures. The stresses imparted by sudden changes in the velocity of super heavy vehicles can potentially jeopardize the longevity of the pavements structures and result in premature failure of transportation facilities. Therefore, analysis of acceleration and deceleration of the SHL vehicles should be an integral component in risk management studies of overload corridors.
\end{abstract}

Keywords: Super Heavy Load (SHL), Overload Corridors, Pavement Service Life, Acceleration and Deceleration Forces, Finite Element Method

\section{INTRODUCTION}

Recent traffic trends and permit issuance records indicate significant demands for operation of the Super Heavy Load (SHL) vehicles in the energy sector and overload corridors of Texas. This is primarily due to the recovery of the state's economy, as well as drastic improvements in freight transportation in overweight corridors. Essentially, movement of the SHL vehicles is 
attributed to a variety of economic activities such as energy production, freight, and transportation of heavy machinery and equipment such as well servicing units, power transformers, and electric generators. Despite facilitating the movement of heavy, large, and non-divisible loads, SHL vehicles typically consist of multi-axle trailer units that weigh several times of the permissible weight limits set forth by regulatory agencies. Operation of these nonconventional vehicles with heavy tires and complex axle arrangements, has been a major source of damages for pavement structures in the network.

The detrimental impact of SHLs on transportation infrastructure is even more pronounced with consideration of the acceleration and deceleration forces applied to the roadway structures. Evidently, this is an ongoing statewide challenge for design agencies across the nation and worldwide, with limited precedence in the literature. Therefore, there is an urgent need to properly assess the distresses imparted by SHL vehicles at multiple loading scenarios, such as sudden acceleration and deceleration, with consideration of seasonal effects on material properties, and type of the pavement facility in the impacted networks. The results can potentially provide insights on the Pavement Life Reduction (PLR) studies, and provide means to improve pavement design protocols.

Several researchers studied different methodologies to evaluate the service life of pavements subjected to SHL vehicle operations. Dong and Huang (2013) evaluated the detrimental impacts of SHLs on pavements in Tennessee through field measurements of the pavement responses. Based on field measurements of the pavement surface deflection, the authors concluded that the evaluated SHLs did not cause considerable deformation on the studied flexible pavement. Chen et al. (2013), using numerical simulations, investigated the consumption of pavement service life due to movement of a SHL vehicle in Louisiana. The researchers converted the SHL axles to equivalent number of standard axles to characterize the loading conditions in their analysis. The authors in turn used this information to estimate the costs associated with the pavement damages. In a recent study, Hajj et al. (2018) developed a mechanistic framework to investigate the impacts of the SHLs on pavements on a case-bycase basis. The researchers used records of issued permits as the primary source to characterize the traffic distributions, and loading conditions attributed to SHLs. In the numerical simulation phase, the researchers considered uniform distribution of contact stresses over a circular loaded area.

Several researchers investigated the influence of acceleration and deceleration of the conventional vehicles on the tire-pavement contact stresses (Wang et al., 2012, and $\mathrm{Hu}$ et al., 2017, Satvati et al., 2021). Based on the numerical simulations, the authors reported that decelerating (braking) vehicles can result in higher contact stresses and therefore higher pavement responses under sudden braking conditions.

The majority of the previous research studies for predicting the pavement service life either rely on the general observations and measurements made in the field, or are based on limited sections and data points. The simplifying assumptions such as the use of permit records in lieu of field data collection, limitation of type of pavement facilities in the study, overlooking the influence of seasonal variation material properties, and unique characteristics of the pavement structure in each location can potentially jeopardize the accuracy and reliability of the damage quantification and remaining life analyses of pavement facilities. Another anomaly persistent in the literature pertains to unrealistic simulation of the tire-pavement contact stresses using uniformly distributed load, rather than considering the non-uniform distribution of the contact stresses. Relying on such simplifying assumptions can be detrimental to the accuracy of the analysis of structural impacts of SHL vehicles with demanding loading conditions. In addition to the elaborated shortcomings, acceleration and deceleration of the non-conventional SHLs with taxing loading conditions, as well as their impacts on the pavement service life is often overlooked in the literature.

The highlighted issues and concerns were the motivations for our research team to devise a protocol study to assess the influence of acceleration and deceleration of SHL vehicles on the imparted damages and loss of service life of pavements for several Farm-to-Market (FM) roads, State Highways (SHs), and US Highways in ten overload corridors. The developed mechanistic framework, accounts for the demanding loading conditions attributed to SHLs, 
site-specific Axle Load Spectra (ALS) databases, various acceleration/deceleration patterns, realistic tire-pavement interactions, and unique features of pavement facilities in the network.

\section{RESEARCH METHODOLOGY}

Figure 1 shows the flowchart of the proposed procedure for the mechanistic characterization of the loss of pavement service life due to acceleration and deceleration of the SHL vehicles. As illustrated in the figure, the proposed analysis framework consisted of three main segments including: (1) characterization of the traffic loading conditions and pavement material properties from field testing, (2) quantification of damages imparted on the pavements using 3D FE modeling, and (3) prediction of the service life of pavements.

To characterize the input parameters, initially, the pavement layer properties were obtained from analyzing the Ground Penetrating Radar (GPR) and Falling Weight Deflectometer (FWD) field data. The pavement design plans were further instrumental in validating or supplementing the information on the material properties, and layer configurations. Subsequently, the authors deployed Portable Weigh-In-Motion (P-WIM) devices to collect sitespecific ALS during summer and winter months, for accurate characterization of traffic in ten representative sites. GVWs, vehicle classifications, axle load distributions, axle weights, axle configurations, and wheel loads were the most relevant traffic information that were extracted from the ALS databases. The authors further developed a procedure for routine measurements of tire pressure and tire footprint in the calibration process of P-WIM devices to achieve an accurate account of the loading conditions. Additionally, the research team conducted an extensive data mining on the permit records and SHL vehicle plans to complement the information on the axle and tire characteristics of the SHLs operating in Texas overload corridors.

Subsequently, the site-specific pavement layers properties and the traffic loading information were in turn incorporated into a 3D FE program for determination of the critical pavement responses under the SHL tires at various rolling conditions, i.e., steady rolling, acceleration, and deceleration (braking). The calculated pavement responses were further incorporated into the mechanistic procedure for pavement damage quantification described in Morovatdar et al. (2020a) to determine the corresponding Equivalent Axle Load Factors (EALFs) tailored towards the specific SHL axle, roadway type, and season of the year.

As stated earlier, the last stage of the analysis pertains to characterization of the influence of tire rolling condition of the SHLs on the pavement service life. To accomplish this, the authors initially incorporated the projected Equivalent Single Axle Load (ESAL) values over a 20-year design life for three evaluated loading scenarios, i.e., ESAL (steady rolling), ESAL (acceleration), and ESAL (deceleration), to characterize the traffic in numerical simulations for further comparison purposes. The cumulative 18-kip (8.16 tons) ESAL values were calculated from Equation 1 as:

$$
E S A L=\sum_{i=1}^{m}(E A L F)_{i} n_{i}
$$

where, $(E A L F)_{i}$ represents the calculated EALF value for $i^{\text {th }}$-axle; and $n_{i}$ is the projected number of passes of $i^{\text {th }}$-axle load group during the design period. As shown in Equation 1, pavement damage equivalency factors, calculated at the second stage of the analysis, are integral components for accurate characterization of the accumulated pavement damages. The number of axle load repetitions were also derived from the site-specific ALS databases. Then, the EALF values for each axle type and axle weight were multiplied by the projected number of load repetitions to calculate the projected ESAL values considering the design life of pavements.

Ultimately, the research team incorporated the ESAL values into the ME pavement design software for comparative analysis of service of life of pavements for the aforementioned three sets of traffic data. Climatic data from an adjacent weather station, as well as performance 
criteria limits, were also assigned using the agency defined thresholds. The analysis results were contrasted with the steady rolling conditions which serve as the benchmark in this study. Therefore, the difference between the service life analyses represents the reduction of the service life associated with the acceleration/deceleration of the SHL vehicles. Additionally, "pavement life reduction" index was calculated to provide a quantitative measure of the severity of the distresses imparted by acceleration/deceleration of the SHL vehicles in the network as:

$$
\text { Pavement Life Reduction }(\mathrm{PLR}) \text { Index }=\frac{P L_{\text {steady } \text { rolling }}-P L_{\text {acceleration } / \text { deceleration }}}{P L_{\text {steady }} \text { rolling }} \times 100
$$

where, $P L_{\text {steady rolling }}, P L_{\text {acceleration, }}$ and $P L_{\text {deceleration }}$ represent the expected pavement life considering the steady rolling, acceleration, and deceleration conditions of the SHL tires, respectively.

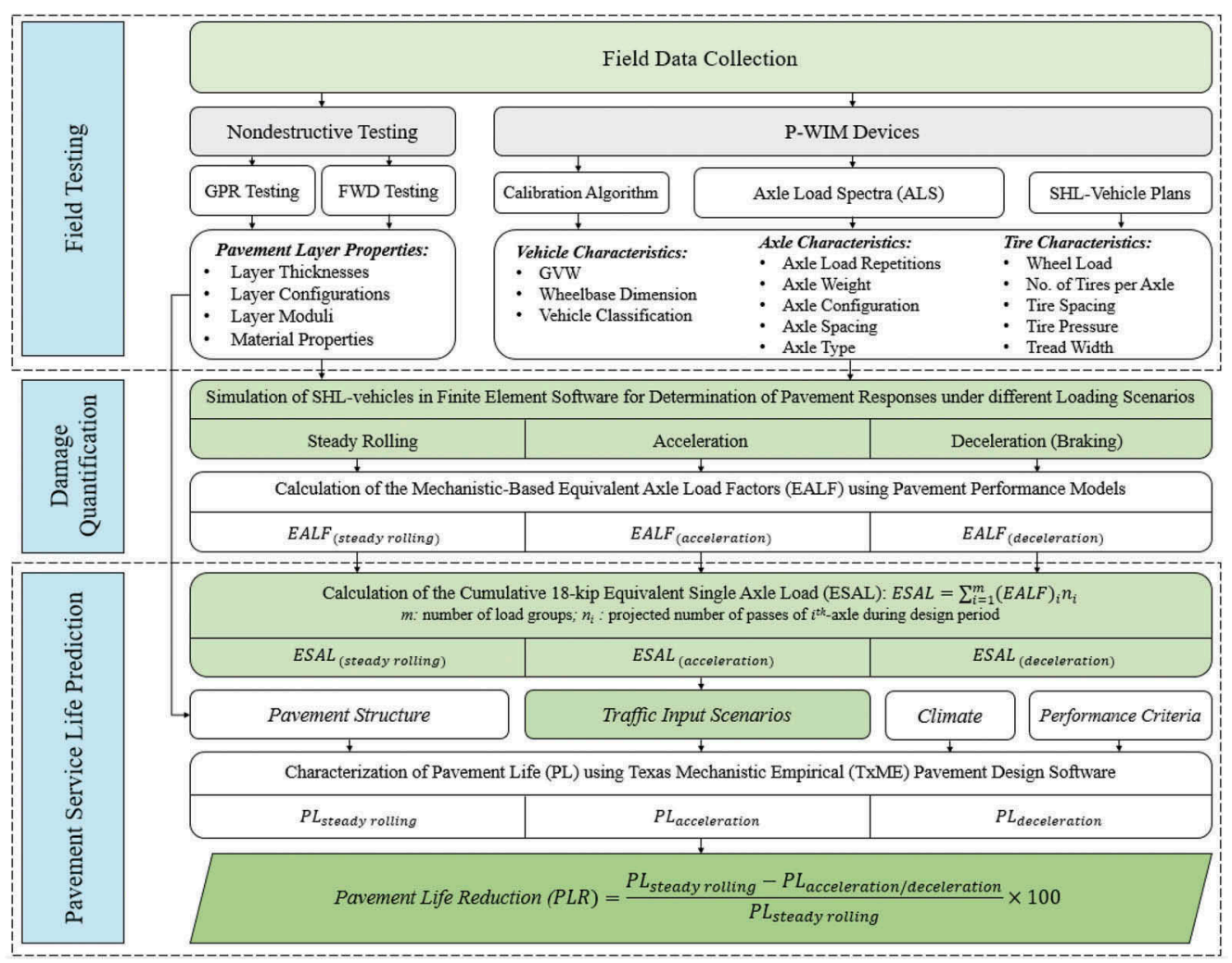

Figure 1. Flowchart for the proposed mechanistic approach for the quantification of the pavement life reduction (PLR) due to acceleration and deceleration of SHL vehicles.

\subsection{Field data collection}

The site-specific information was collected for ten representative sites strategically distributed throughout the overload corridors in Texas including Corpus Christi, Laredo, Yoakum, and San Antonio Districts. The authors deployed GPR and FWD to the field for the determination of the layer profile and back-calculation of layer moduli in representative pavement sections, as shown in Figures 2(a) and 2(b). Analyzing the GPR data validated by pavement design plans showed that representative pavement sections in FM roadways consisted of less 
robust pavement profile with thin structure, compared to more robust pavement structures of SH and US highways (Morovatdar et al., 2019, 2020b, 2020c). The authors also developed field testing plans for FWD testing in two different seasons (summer and winter), to properly evaluate the effect of environmental parameters on the back-calculated layer modulus of pavement layers.

The P-WIM devices were also deployed in selected FM, SH, and US roadways to collect the site-specific traffic information. The P-WIM units were temporarily installed at each location and were left to continuously record traffic information for at least two weeks in each site. The process was repeated for both summer and winter times to capture the seasonal effect of traffic variations. Figure 2(c) shows the piezo-electric sensors inserted into specialized pocket tapes, as well as the data acquisition system, as principal components of the P-WIM units deployed for characterization of the ALS in the field.

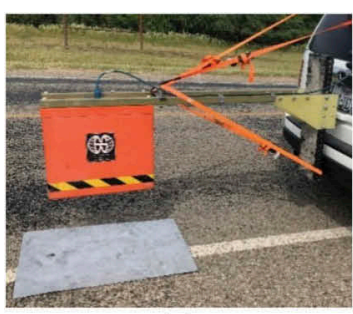

(a)

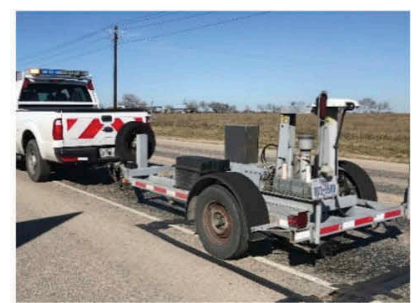

(b)

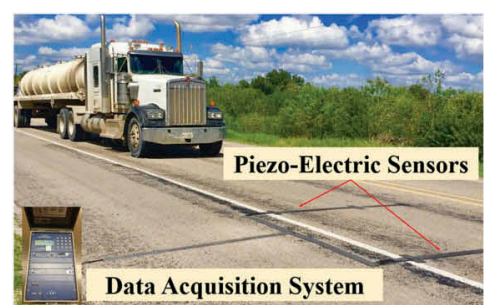

(c)

Figure 2. Data collection effort (a) GPR testing, (b) FWD testing, (c) P-WIM deployment.

The analysis of the ALS indicated high number of SHL operations in the surveyed network. Table 1 shows the SHL axle ranges for different axle group types, based on field data. Legal and the maximum permissible weight limits for trucks operating in Texas are also provided for comparison purposes. Axle weights heavier than legal limits are primarily considered as overweight, while the axles that exceed the maximum allowable weight limits are essentially regarded as super heavy loads. As evidenced in Table 1, the maximum recorded axle loads substantially deviate from the maximum permissible limits. The heaviest axle load was found to be 63.5 tons for quad axles, as recorded during the data collection in SH 123 in Corpus Christi. Relevant information on extensive field data collection efforts, and the synthesized findings attributed to all ten representative sites, are provided in Ashtiani et al. (2019).

Table 1. Super Heavy Loads Collected by P-WIM Devices.

\begin{tabular}{llll}
\hline & \multicolumn{2}{l}{ Axle Weight, $l b .($ tons $)$} & \\
\cline { 2 - 4 } Axle Type & $\begin{array}{l}\text { Legal Weight Limit } \\
\text { (OW Threshold) }\end{array}$ & $\begin{array}{l}\text { Maximum Permissible } \\
\text { (SHL Threshold) }\end{array}$ & $\begin{array}{l}\text { Maximum Recorded Axle } \\
\text { Weights in Field Trials }\end{array}$ \\
\hline Single Axle & $20,000(9.1)$ & $25,000(11.3)$ & $42,000(19.1)$ \\
Tandem Axle & $34,000(15.4)$ & $46,000(20.8)$ & $120,000(54.4)$ \\
Tridem Axle & $42,000(19.1)$ & $60,000(27.2)$ & $114,000(51.7)$ \\
Quad Axle & $50,000(22.7)$ & $70,000(31.8)$ & $140,000(63.5)$
\end{tabular}




\subsection{Finite element modeling}

In this study, ABAQUS FE program was used for determination of the SHL-induced pavement responses under various tire rolling conditions, with considerations of the viscoelastic nature of Asphalt Concrete (AC) layer, complex traffic loading conditions, and tire-pavement interactions. Subsequent sections provide the relevant information on the 3D FE models developed in this study for simulation of the pavement structures, material behavior models, SHL tires at acceleration/deceleration conditions, and tire-pavement interactions. Detailed information on input parameters including material models, material properties, and traffic characteristics are provided in Ashtiani et al. (2019).

\subsubsection{Simulation of the pavement structure}

Based on the nondestructive testing results, pavement structures consisting of AC, base, and subgrade layers were modeled in the software. The site-specific information on the pavement layer thicknesses, layer configurations, as well as the layer moduli were incorporated into the developed FE code to simulate the structural characteristics of the representative pavement sections. The viscoelastic properties of the AC surface layers were also incorporated in the analysis to account for the influence of temperature and frequency of loading in this study.

\subsubsection{AC viscoelastic behavior modeling}

The structural capacity of multi-layer pavement structures is not monolithic under different loading frequencies and different temperatures (Muzenski et al. 2020, Salimi et al. 2019; 2021). This is primarily attributed to the time- and temperature-dependent behavior of the viscoelastic AC layers. In this study, the viscoelastic properties of the AC surface layers were incorporated in the analysis to account for the influence of temperature and frequency of loading. Hence, the generalized Maxwell model was used to characterize the viscoelastic properties of the AC layers through FE numerical simulations in this study, as presented in the following equations:

$$
\begin{gathered}
s=\int_{-\infty}^{t} 2 G(t-\tau) \frac{d e}{d \tau} d \tau \\
p=\int_{-\infty}^{t} K(t-\tau) \frac{d(\operatorname{tr}[\varepsilon])}{d \tau} d \tau
\end{gathered}
$$

where $s$ is deviatoric stress, $e$ is deviatoric strain, $p$ is volumetric stress, $\operatorname{tr}[\varepsilon]$ is trace of volumetric strain, $t$ is relaxation time, and $K$ and $G$ are the bulk and shear moduli of AC layer, respectively. Bulk $(K)$ and shear $(G)$ moduli of AC were obtained from laboratory dynamic modulus tests. The Prony series were then used to calculate the corresponding modulus values in time domain to satisfy the FE software requirements, as indicated through Equations 5 and 6:

$$
\begin{aligned}
& G(t)=G_{0}\left[1-\sum_{i=1}^{n} G_{i}\left(1-e^{-t / \tau_{t}}\right)\right] \\
& K(t)=K_{0}\left[1-\sum_{i=1}^{n} K_{i}\left(1-e^{-t / \tau_{t}}\right)\right]
\end{aligned}
$$

where $G_{0}$ and $K_{0}$ are instantaneous shear and volumetric elastic moduli; and $G_{i}, K_{i}$, and $\tau_{i}$ are the Prony series parameters. The information on the dynamic modulus tests, as well as the Prony series parameters, were obtained from a relevant study conducted by $\mathrm{Hu}$ et al. (2017).

\subsubsection{Simulation of the SHL tire}

The tire element, including tire ribs and grooves, was explicitly modeled with consideration of the characteristics and tread patterns of SHL-vehicle tires operating in Texas overload corridors. Figure 3 illustrates the cross-sectional and 3D views of the modelled tire. Based on the 
information extracted from the review of the SHL vehicle plans and permit records in Texas, it was found that the SHL tire's height ranged from 28 in. to $35 \mathrm{in}$. $(71 \mathrm{~cm}$ to $88 \mathrm{~cm})$, while tread width of the tires varied between 6 and $10 \mathrm{in}$. $(15 \mathrm{~cm}$ and $25 \mathrm{~cm})$, depending on the tire configuration and axle assembly of different SHL vehicles. The elastic material properties of the tire element were also defined based on the relevant information provided in the literature (Wang et al., 2012).
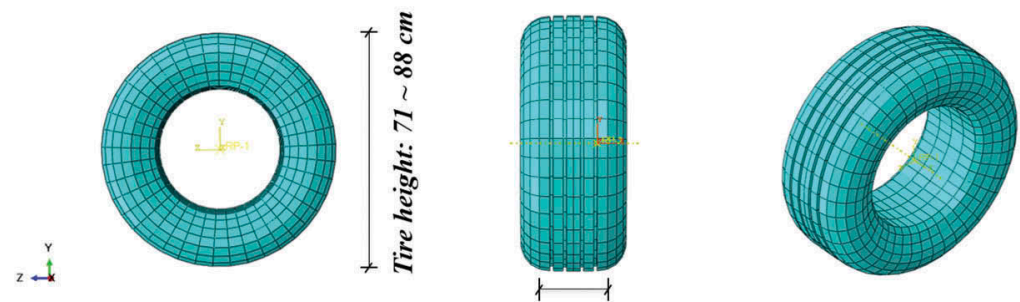

Tread width: $15 \sim 25 \mathrm{~cm}$

Figure 3. Simulation of the SHL tire using 3D FE models.

\subsubsection{Tire-pavement contact stresses}

Realistic simulation of the tire-pavement contact stresses is of greatest importance for accurate assessment of the SHL-induced pavement responses. Figure 4 provides an example associated with the simulated tire-pavement contact stresses under a SHL tandem-axle with 120 kips (54.4 tons) axle weight and 15 kips (6.8 tons) load magnitude on each individual tire. As shown in Figure 4, the simulated tire element in this study properly captured the non-uniform distribution of the tire-pavement contact stresses under each individual tire rib. The characterized convex-shape distribution pattern of contact stresses along the tire contact length is consistent with the experimental measurements and observations made by previous researchers (Douglas et al., 2000; Wang and Al-Qadi 2009). Based on the contract stress distributions provided in Figure 4, the maximum calculated contact stress was $180 \mathrm{psi}(1,241 \mathrm{kPa})$ within the middle tire ribs; while, the average value was calculated as $125 \mathrm{psi}(861 \mathrm{kPa})$. This average value for contact stresses is in line with our research team field measurements of tire pressure in Texas (Ashtiani et al., 2019).

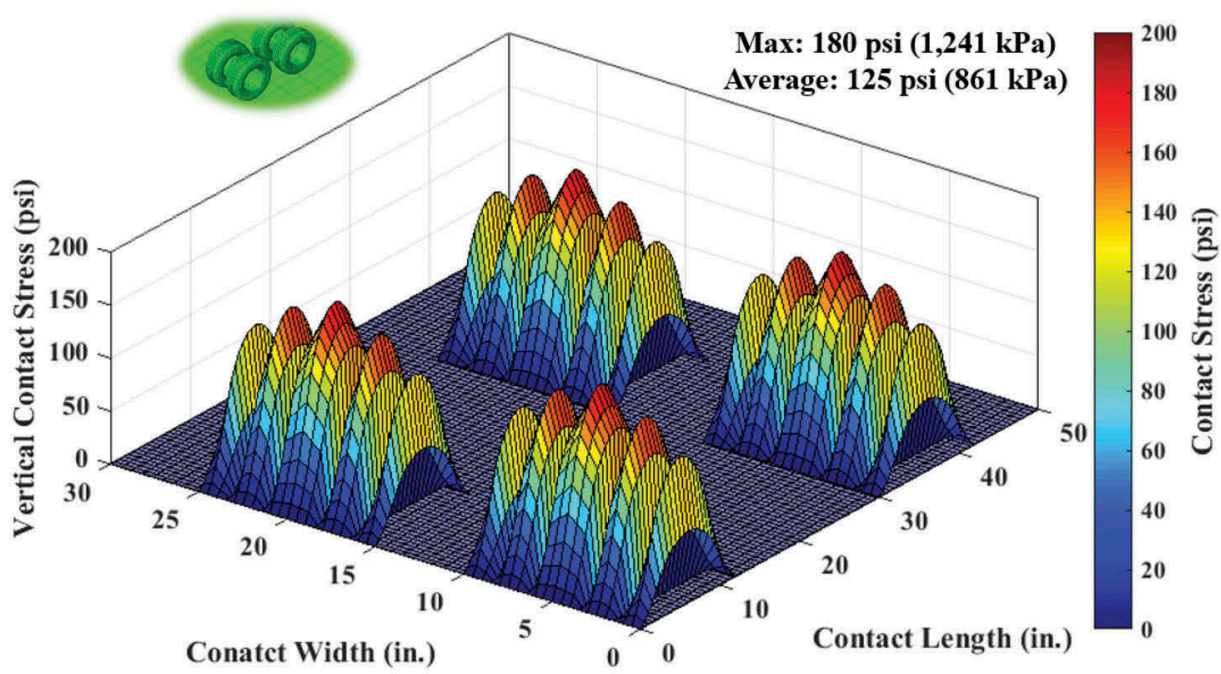

Figure 4. Non-uniform tire-pavement contact stresses under a 120-kips (54.4 tons) SHL tandem-axle. 
It should be also noted that a friction coefficient of 0.5 , as recommended by Wang et al. (2012), was incorporated into the numerical simulations to account for the frictional effects at the tire-pavement interface. To accomplish this, the penalty method, as well as the Coulomb friction law were used to simulate the normal and tangential interactions, respectively.

\subsubsection{Various tire rolling conditions}

The authors used relevant user subroutines and dynamic analysis offered in ABAQUS to simulate the tire rolling procedure. Translational speed $(v)$, as well as the corresponding rotational speed $(\omega)$, were assigned to the SHL-tires to simulate the steady rolling condition. The kinematic coupling constraint method was also used to allow movement of the tires along traffic direction. Furthermore, translational acceleration (a) and rotational acceleration $(a)$ were separately defined to account for the additional driving/braking torques applied on the SHL tire under acceleration/deceleration conditions. Relatively low vehicle speed (i.e., $v=32 \mathrm{kph}$, $\omega=11.7 \mathrm{rad} / \mathrm{s}$ ) to account for slow-moving nature of SHLs, and different acceleration/deceleration levels (i.e., $a=0.6 \mathrm{~m} / \mathrm{s}^{2}, 1.5 \mathrm{~m} / \mathrm{s}^{2}, 3.5 \mathrm{~m} / \mathrm{s}^{2} ; a=1.2 \mathrm{rad} / \mathrm{s}^{2}, 2.9 \mathrm{rad} / \mathrm{s}^{2}, 5.8 \mathrm{rad} / \mathrm{s}^{2}$ ) were incorporated into the comprehensive sensitivity analyses conducted in this study.

\section{FIELD DATA ANALYSIS AND RESULTS}

\subsection{Influence of SHL acceleration/deceleration on damage factors}

Pavement damage factors, namely EALFs, allow for the quantification of the pavement damages per pass relative to a standard 18-kip single axle. The EALF values attributed to the various tire rolling conditions (i.e., steady rolling, acceleration, and deceleration) were contrasted with each other for comparison purposes in Figure 5. The results are specifically tailored towards the SHL tandem-axle load group in State Highways. As evidenced in the plot, in all axle weights, decelerating (braking) tires had the highest damage factors, followed by accelerating and ultimately steady rolling tires. This is primarily attributed to the fact that when a tire is under deceleration/acceleration conditions, the resulting longitudinal tire-pavement contact stresses significantly increase due to the frictional interactions at the tire-pavement interface. Such excessive contact stresses lead to development of the significant shear stresses in the pavement structure, which in turn translates into higher shear rutting in pavements. Consequently, damage assessment and service life analysis protocols should properly account for deceleration and acceleration of the SHLs and their detrimental impacts on the longevity of pavement structures. It is also noted that similar EALF trends were observed from the numerical simulations for various axle types and roadways evaluated in this research study.

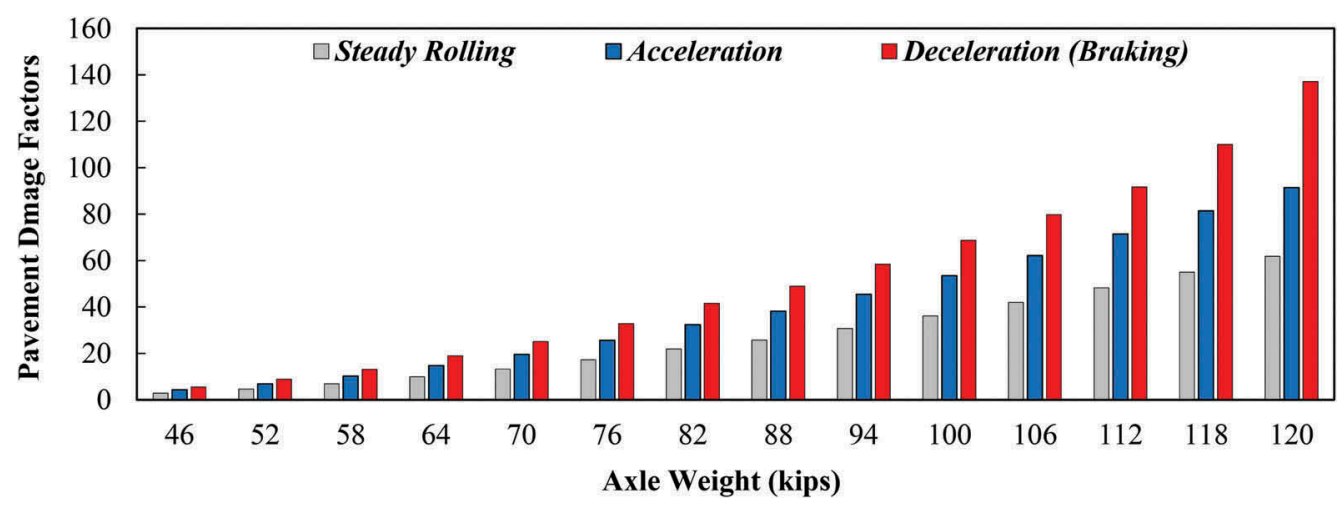

Figure 5. Pavement damage factors for various tire rolling conditions of SHL tandem-axles. 


\subsection{Influence of SHL acceleration/deceleration on pavement service life}

Figure 6 provides the results pertaining to one of the heavily trafficked sections, SH123 in Corpus Christi, as an example to showcase the analysis protocol developed in this study for mechanistic quantification of the loss of pavement service life. The results are based on frequent application of SHL vehicles as captured by P-WIM devices. The plot shows the rutting performance over a 20 -year design period for SH123, considering three evaluated tire rolling scenarios. Based on the internal distress calculation models in mechanistic analysis, and steady-rolling based damage factors, it takes 168 months for SH123 to develop 0.5 in. $(12.7 \mathrm{~mm})$ of rut depth. However, if the acceleration-based damage factors were incorporated into the analysis framework, it takes 135 months to develop 0.5 in. $(12.7 \mathrm{~mm})$ of cumulative rut depth. In other terms, inclusion of acceleration patterns of SHL vehicles in SH123 can essentially consume $(168-135=33)$ months of the service life of this pavement section. Additionally, deceleration considerations of the SHL vehicles in SH123 can result in 63 months reduction in its pavement service life. Consequently, the analysis of the loss of pavement service life for SH123 indicated that acceleration and deceleration of the SHL vehicles operating in this roadway can impart significant PLRs as high as $20 \%$, and $38 \%$, respectively.

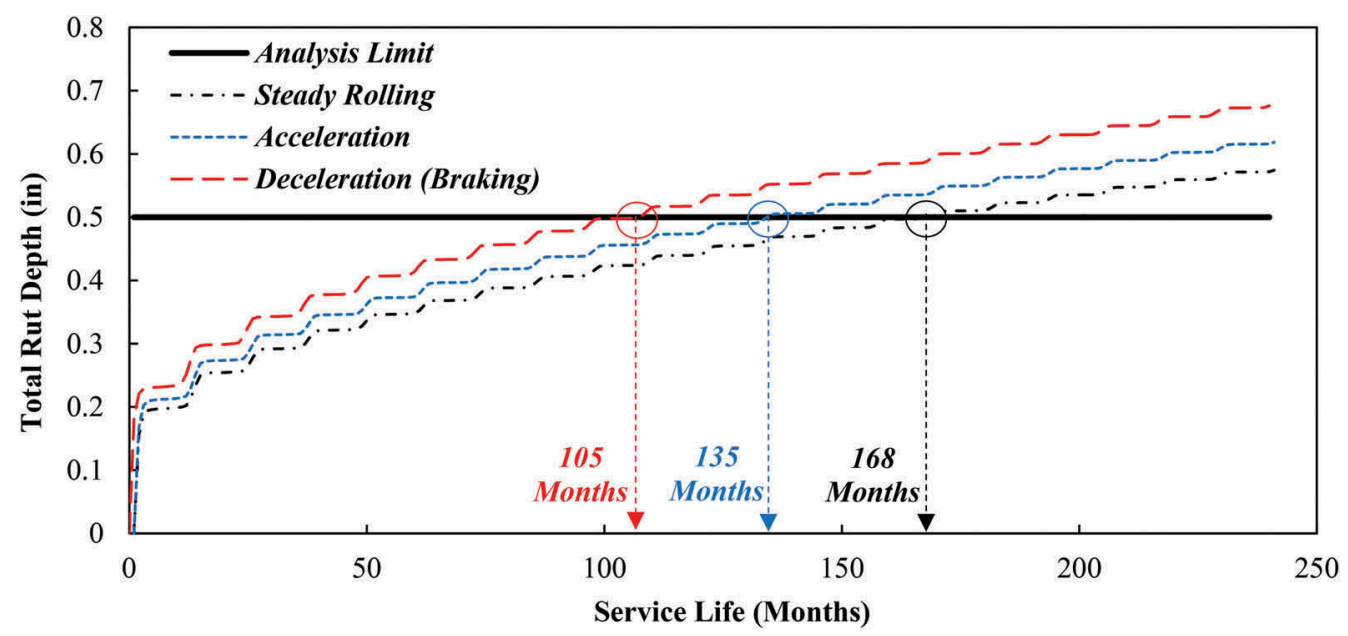

Figure 6. Pavement life reduction for SH123 due to acceleration and deceleration of SHLs.

\subsection{Pavement life reduction for various roadway types}

Figure 7 illustrates the results associated with the pavement life reduction for various roadway types (i.e., FM, SH, and US highways) due to acceleration and deceleration of SHL vehicles. According to the sensitivity results of multiple loading scenarios, FM roadways with less robust pavement structures were found to be more sensitive to the acceleration and deceleration of SHL vehicles, compared to the representative pavement sections in SH and US highways with higher structural capacity. This highlighted the importance of the pavement profile on the analysis of the acceleration and deceleration of the SHL vehicles.

The results illustrated in Figure 7 also underscored the significance of sudden variations in SHL vehicle speed. According to the results provided in Figure 7, frequent acceleration of SHL vehicles in US, SH, and FM roadways can potentially consume $18 \%, 20 \%$, and $26 \%$, respectively, of service life of their pavement sections; while, deceleration of such vehicles can result in PLRs as high as $32 \%, 38 \%$, and $42 \%$ for US, SH, and FM roadways, respectively. Therefore, pavement structures servicing overload corridors, particularly at 
intersections or stop-go sections with significant frequencies of braking, can potentially experience excessive distresses due to absence of a protocol to account for horizontal and tangential forces exerted during acceleration and deceleration scenarios during the pavement design process.

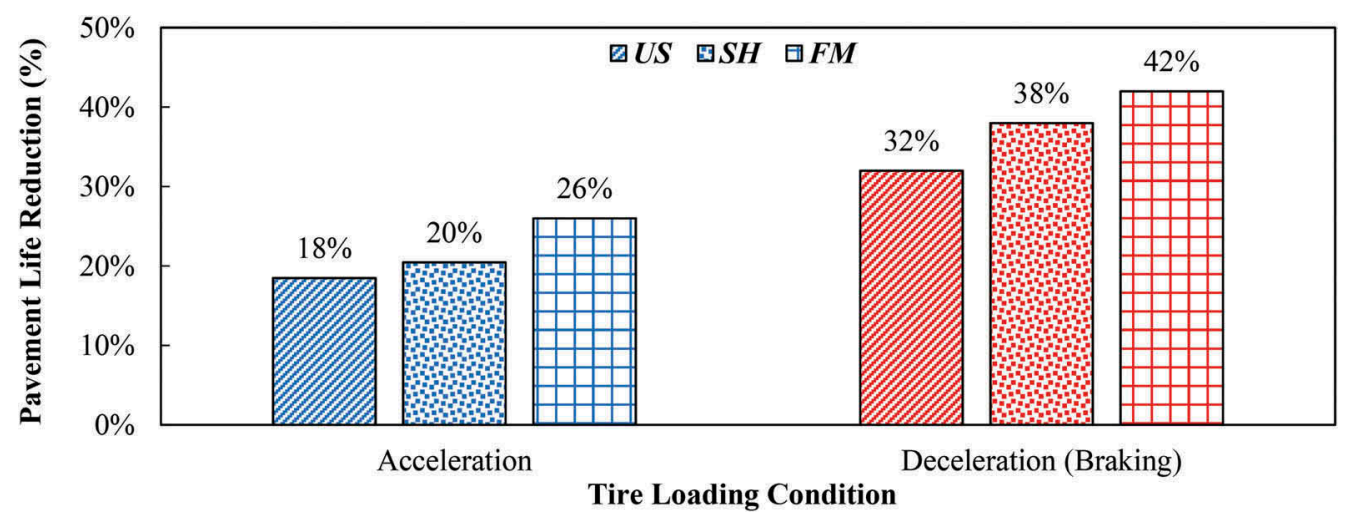

Figure 7. PLR due to acceleration/deceleration of SHLs for different roadway types.

\section{SUMMARY AND CONCLUSIONS}

In this study, a mechanistic framework was developed for characterization of the loss of pavement service life due to acceleration and deceleration of the SHL vehicles. The developed framework accounts for the taxing stress paths imparted by SHLs, tire-pavement interactions, unique features of transportation systems, and the environmental factors for accurate assessment of the pavement service life. The numerical simulation results in this study underscored the significance of acceleration and deceleration loading scenarios for accurate assessment of SHLs in overload corridors. The sensitivity analysis provided in this study showed that accelerating and decelerating heavy vehicles can impart substantially higher damages on the pavement structures, compared to the same vehicle travelling at constant speed. This is primarily attributed to the dynamic nature of the analysis, and the added horizontal and tangential forces associated with speeding and braking actions.

Further mechanistic analysis of the pavement service life in this study showed that deceleration of the SHL vehicles results in higher levels of PLR, compared to the acceleration of these vehicles under similar conditions. Loss of pavement service life was more substantial in FM roadways with less robust pavement structures, compared to $\mathrm{SH}$ and US highways. The post-processed results for the representative pavement sections in this study revealed that acceleration and deceleration of the SHL vehicles operating in FM roadways can impart PLR as high as $26 \%$ and $42 \%$, respectively.

The results of this study highlighted the significance of acceleration/deceleration of SHL vehicles and their impact on the accumulated damages imparted on the pavement sections. Therefore, pavement structures, particularly at intersections, junctions, or stop-go sections, can potentially experience excessive distresses due to absence of a protocol to account for the detrimental impacts of acceleration and deceleration scenarios during the pavement design process. Consequently, analysis of acceleration and deceleration of SHL vehicles should be an integral component in risk management studies of pavement facilities servicing the overload corridors. 


\section{REFERENCES}

Ashtiani, R. S., Morovatdar, A., Licon, C., Tirado, C., Gonzales, J., and Rocha, S., 2019. Characterization and Quantification of Traffic Load Spectra in Texas Overweight Corridors and Energy Sector Zones. No. FHWA/TX-19/0-6965-1.

Chen, X., Lambert, J. R., Tsai, C., and Zhang, Z., 2013. Evaluation of Superheavy Load Movement on Flexible Pavements. International Journal of Pavement Engineering, 14(5), 440-448.

Dong, Q., and Huang, B., 2013. Field Measurement of Pavement Responses under Super Heavy Load.

Douglas, R.A., Woodward, W.D.H., and Woodside, A.R., 2000. Road Contact Stresses and Forces under Tires with Low Inflation Pressure. Canadian Journal of Civil Engineering, 27, 1248-1258.

Hajj, E. Y., Siddharthan, R. V., Nabizadeh, H., Elfass, S., Nimeri, M., Kazemi, S. F., ... and Piratheepan, M., 2018. Analysis Procedures for Evaluating Superheavy Load Movement on Flexible Pavement. Report No. FHWA-HRT-18-049, FHWA, Washington, DC.

Hu, X., Faruk, A. N., Zhang, J., Souliman, M. I., and Walubita, L. F., 2017. Effects of Tire Inclination (Turning Traffic) and Dynamic Loading on the Pavement Stress-Strain Responses using 3-D Finite Element Modeling. Pavement Research and Technology, 10 (4).

Morovatdar, A., Ashtiani, R. S., Licon, C., and Tirado, C., 2019. Development of a Mechanistic Approach to Quantify Pavement Damage using Axle Load Spectra from South Texas Overload Corridors. In GeoStructural Aspects of Pavements, Railways, and Airfields Conference (GAP 2019), Colorado Springs, CO, USA.

Morovatdar, A., Ashtiani, R. S., Licon, C., Tirado, C., and Mahmoud, E., 2020a. Novel Framework for the Quantification of Pavement Damages in the Overload Corridors. Transportation Research Record 2674, no. 8, 179-191.

Morovatdar, A., Ashtiani, R. S., and Licon Jr, C., 2020b. Development of a mechanistic framework to predict pavement service life using axle load spectra from Texas overload corridors. In International Conference on Transportation and Development 2020 (pp. 114-126). Reston, VA: American Society of Civil Engineers.

Morovatdar, A., \& Ashtiani, R. S. (2020c). Evaluation of pavement service life reduction in overload corridors. In Advances in Materials and Pavement Performance Prediction II: Contributions to the 2nd International Conference on Advances in Materials and Pavement Performance Prediction (AM3P 2020), 27-29 May, 2020, TX, USA (p. 211).

Muzenski, S., Flores-Vivian, I., Farahi, B., \& Sobolev, K. (2020). Towards ultrahigh performance concrete produced with aluminum oxide nanofibers and reduced quantities of silica fume. Nanomaterials, 10(11), 2291.

Salimi, K., \& Ghazavi, M. (2019). Soil reinforcement and slope stabilisation using recycled waste plastic sheets. Geomechanics and Geoengineering, 1-12.

Salimi, K., Cerato, A. B., Vahedifard, F., \& Miller, G. A. (2021). Tensile Strength of Compacted Clays during Desiccation under Elevated Temperatures. Geotechnical Testing Journal, 44(4).

Satvati, S., Nahvi, A., Cetin, B., Ashlock, J. C., Jahren, C. T., \& Ceylan, H. (2021). Performance-based economic analysis to find the sustainable aggregate option for a granular roadway. Transportation Geotechnics, 26, 100410.

Wang, H. and Al-Qadi, I.L., 2009. Combined Effect of Moving Wheel Loading and Three-Dimensional Contact Stresses on Perpetual Pavement Responses. Transportation research record, No. 2095. Washington, DC: TRB, 53-61.

Wang, H., Al-Qadi, I. L., and Stanciulescu, I., 2012. Simulation of Tyre-Pavement Interaction for Predicting Contact Stresses at Static and Various Rolling Conditions. International Journal of Pavement Engineering, 13(4), 310-321. 